\title{
Superstructure and Superimposed Fiber Bragg Gratings Fabricated through Small Spot Direct UV Writing
}

\author{
Senta L. Jantzen ${ }^{1, *}$, Devin H. Smith ${ }^{1}$, Rex H. S. Bannerman ${ }^{1}$, Paolo L. Mennea ${ }^{1}$, \\ Lewis J. Boyd ${ }^{2}$, Peter G. R. Smith ${ }^{1}$ and Christopher Holmes ${ }^{1}$ \\ 1 Optoelectronics Research Centre, University of Southampton, SO17 1BJ, UK \\ 2 Parker Aerospace, Parker Hannifin Corporation, 2510 The Quadrant, Bristol, BS32 4AQ, UK \\ * Corresponding author: S.Jantzen@soton.ac.uk
}

\begin{abstract}
We demonstrated the fabrication of a record number of over 40 overlaid fiber Bragg gratings. We compared two different fabrication techniques: consecutively superimposed and digitally superstructured gratings and analysed the evolving AC refractive index change. (C) 2020 The Author(s)
\end{abstract}

\section{Introduction}

Fiber Bragg gratings (FBGs) are most often used for sensing, but they also find commercial application for network identification [1]. Using Bragg gratings to write unique network addresses into fibers to remotely interrogate optical lines in fiber networks can facilitate network management. By defining the Bragg wavelengths outside of the data transmission range, interference with the data signal is avoided. To decrease the effect of local temperatures changes, it can be beneficial to write those gratings at the same physical location. This also allows a smaller device footprint.

The maximum number of overlaid gratings which has been reported in literature is 16 [2]. Due to a saturation of the "AC" index change, the number of gratings that can be written on top of each other is limited.

This work presents two different techniques for overlaying a record of over 40 Bragg gratings and analyses the development of refractive index for consecutively written gratings.
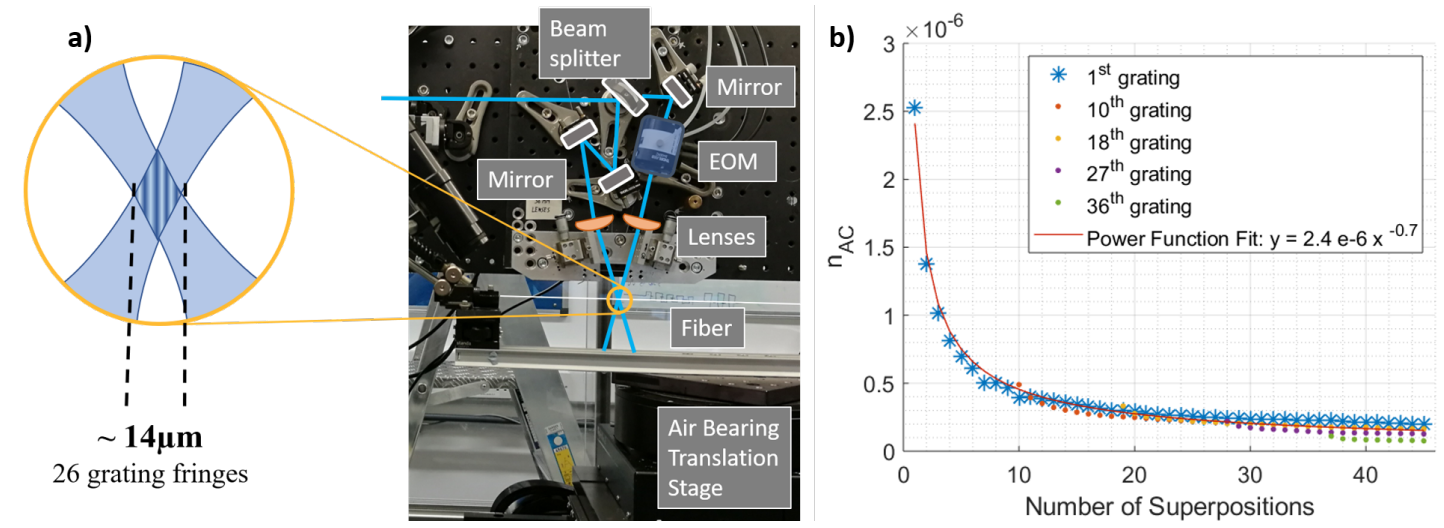

Fig. 1. a) Small spot direct UV writing set up. b) Development of the "AC" part of the refractive index for a range of gratings during evolving superposition.

\section{Fabrication and Results}

Small Spot Direct UV Writing (SSDUW) has been used to fabricate the gratings with a length of $200 \mathrm{~mm}$ in photosensitive polarisation maintaining fiber (PS-PM980). This technique uses a small interference laser spot, which is created by splitting up and recombining two beams in the core of a fibre to inscribe Bragg gratings [3]. The fibre-coating is removed and the fibre is clamped on both ends into fiber holders, which are mounted on an air-bearing translation stage. The fiber is then tracked and translated under the interference beam, with exposes the core. Fig. 1 a) displays the set up with an inset of the $14 \mu \mathrm{m}$ interference sport, which consists of approximately 26 grating planes. 
This work demonstrates the fabrication of 45 superimposed gratings, where the gratings are fabricated by consecutive exposure of one physical location as well as 44 superstructure gratings, where a refractive index pattern of multiple superpositioned gratings is digitally designed and written at once.

The superimposed gratings are formed by writing one grating after the other, which allows a spectral analysis of the evolving optical spectrum. A broadband super luminescent source (Amonics ASLD-CWDM-5B-FA) was connected to an optical spectrum analyser and the fiber via a $3 \mathrm{~dB}$ coupler. The change in the "AC" part of the refractive index for a range of gratings during the inscription of consecutive gratings is shown in Fig. 1 b). With an increasing number of superpositions, the photosensitivity of the fiber becomes saturated and the achievable contrast, or "AC" index change decreases with a power function. This implies that there is a fundamental limit on the number of superimposed gratings that can be achieved.

The fabrication of superstructure gratings varies from superimposed gratings such that the refractive index pattern for all gratings is digitally designed, by overlaying sine curves with different frequencies, and all gratings are written at once. The advantage of this approach is that a saturation of photosensitivity should not occur, as the fiber is only exposed once, rather than multiple times. However, with an increased number of gratings, the designed refractive index pattern becomes very fine until the small structures cannot be resolved anymore.

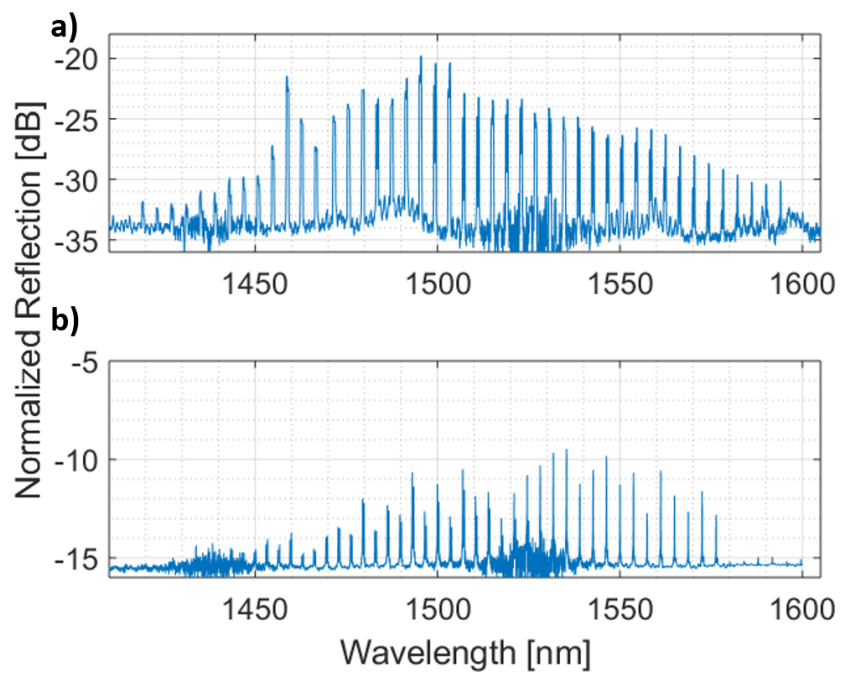

Fig. 2. a) 45 superimposed and b) 44 superstructured fiber Bragg gratings written in PS-PM980.

The spectrum for 45 superimposed and 44 superstructured gratings is shown in Fig. 2 a) and b). Both techniques allow the definition of more than 40 Bragg gratings in a wide wavelength range of almost $190 \mathrm{~nm}$. Both spectra display a smaller grating reflection for short and long wavelengths. This is due to the writing technique, which possesses a characteristic detuning curve.

Further work will investigate the spectral performance for a narrower wavelength range as well as a spectral comparison for grating inscription in hydrogenated fiber. It can be assumed that hydrogen can re-in-diffuse after each grating inscription, making the superimposed grating writing technique the favourable writing approach.

\section{Conclusion}

We demonstrated the fabrication of a record number of more than 40 overlaid gratings using superimposed and superstructure writing. We fabricated those gratings over a broad wavelength range from $1410-1600 \mathrm{~nm}$. We will further investigate the effects of grating definition in hydrogenated fiber, as hydrogen re-in-diffusion may occur with superimposed grating writing.

\section{References}

1. Castiglioni, M., Favero, D. Del., "OLiD : Diamond's FBG Network Monitoring System”, White Paper, 2015.

2. Ibsen, M., Durkin, M. K., Cole, M. J., Laming, R. I., "Sinc-Sampled Fiber Bragg Gratings for Identical Multiple Wavelength Operation", IEEE Photonics Technology Letters, 10(6), 1999.

3. Scholl, S. L., Jantzen, A., Bannerman, R. H. S., Gow, P. C., Smith, D. H., Gates, J. C., Holmes, C. ’Thermal approach to classifying sequentially written fiber Bragg gratings", Optics Letters, 44(3), 2019. 